\title{
Les normes britanniques relatives à l'exposition aux descendants du radon dans les habitations *
}

\author{
G.A.M. WEBB et M.C. O'RIORDAN** \\ (Manuscrit reçu le 23 mai 1988)
}

\begin{abstract}
RÉSUMÉ
Le National radiological protection board (NRPB) a émis un avis officiel sur les normes à adopter en Grande-Bretagne pour le contrôle de l'exposition aux produits de filiation du radon dans les habitations qui existent actuellement et pour la modification des procédures de construction applicables aux habitations futures. Ces normes sont celles recommandées par la Commission internationale de protection radiologique (CIPR), adaptées au contexte britannique. Le NRPB a considèrè notamment la conversion en équivalent de dose efficace des concentrations mesurées en radon et les niveaux de risque impliqués, la comparaison de ces niveaux avec les risques dus à d'autres causes, le nombre d'habitations susceptibles d'être impliquées, leur répartition géographique, les coûts et l'efficacité des mesures à prendre.
\end{abstract}

Le NRPB a retenu pour les habitations actuelles le niveau d'action recommandé par la CIPR, soit $20 \mathrm{mSv}$ par an; par contre, pour les habitations nouvelles, la limite supérieure de $5 \mathrm{mSv}$ par an est inférieure à celle de la CIPR.

\section{ABSTRACT}

The National Radiological Protection Board has issued formal advice on the standards to be adopted in the UK for control of exposures to radon daughters in existing dwellings and for changes in building procedures for future dwellings. The standards are based on those recommended by the International commission on radiological protection but adapted to circumstances in the United Kingdom. The matters taken into account by the Board when formulating its advice, and which are discussed in the paper, include the conversion from measured concentrations of radon to effective dose equivalent and the implied levels of risk, the comparison of these levels with risks from other causes, the numbers of dwellings in which various annual doses are likely to be exceeded, the geographical distribution of these dwellings, the likely costs and effectiveness of various remedial measures and the degree of domestic disruption.

The action level for existing dwellings selected by the Board was the same, $20 \mathrm{mSv}$ per annum, as that recommended by the ICRP, but the upper bound for new dwellings of $5 \mathrm{mSv}$ per annum was lower than that suggested by the ICRP.

- Communication présentée au $7^{\mathrm{e}}$ congrès international de I'IRPA, IRPA 7, Sydney, 10-17 avril 1988

** National Radiological Protection Board (NRPB), Chilton, Didcot, Oxon, OX11 ORQ, Grande-Bretagne. 


\section{INTRODUCTION}

En 1977, le ministère de la santé a donné au NRPB instruction officielle de conseiller les départements ministériels intéressés et les corps constitués, en ce qui concerne la recevabilité des recommandations et des propositions, ainsi que leur application dans le Royaume-Uni, chaque fois que de telles recommandations ou propositions sont publiées par la CIPR, comme par d'autres organisations. Le NRPB a étudié la question de l'exposition au radon dans les habitations; il a émis un avis officiel sur les normes en janvier 1987 [4], accompagné d'un document justificatif [5]. Notre article expose l'essentiel de cet avis, et ce qui en découle.

\section{LES PROJETS DE LIMITATION}

En 1984, la Commission internationale de protection radiologique (CIPR) a publié les principes concernant la limitation de l'exposition du public aux sources naturelles de rayonnement; pour l'essentiel, il s'agissait de l'exposition aux descendants du radon, à l'intérieur des habitations [1]. La CIPR faisait une distinction entre la philosophie des contrôles à appliquer aux habitations existantes et aux habitations futures; elle précisait les critères de limitation concernant les descendants du radon, dans ces deux cas.

Pour les habitations existantes, où toute intervention ne peut être que curative, elle suggérait un niveau d'action correspondant à un équivalent de dose efficace annuel de $20 \mathrm{mSv}$ (concentration en équivalent radon, à l'équilibre (ERE), de $200 \mathrm{~Bq} \mathrm{~m}^{-3}$. Pour ce niveau d'action, on pourrait se limiter à des mesures simples de réduction de l'exposition. $A$ un niveau beaucoup plus élevé, il serait possible d'en arriver à des interventions plus importantes.

Pour les habitations futures, contrôlables au stade de la décision de construction et à celui de la mise en œuvre, la CIPR suggérait une limite supérieure correspondant à un équivalent de dose efficace annuel de $10 \mathrm{mSv}$. Elle avait obtenu cette valeur, principalement en comparant le risque relatif de cancer du poumon aux risques domestiques.

Un peu plus tôt, en 1984, la Commission royale sur la pollution de l'environnement (RCEP) avait examiné la qualité de l'air à l'intérieur des habitations [6]. Elle recommandait d'intervenir dans les habitations existantes où l'équivalent de dose efficace annuel dépasse $25 \mathrm{mSv}$; quoiqu'elle considérât une valeur plus faible de $5 \mathrm{mSv}$ pour les constructions futures, elle était disposée à attendre une décision à venir de la CIPR. Les points de vue des deux commissions étaient donc semblables, en ce qui concerne le concept, bien qu'il existât de légères différences sur les valeurs numériques. Dans sa réponse au rapport de la RCEP, le gouvernement exprima son intention d'attendre l'avis du NRPB avant d'envisager les mesures à prendre. 


\section{LES RISQUES LIÉS AU RADON}

On peut calculer le risque d'induction du cancer du poumon dû à une exposition chronique aux descendants du radon en partant du facteur de risque de $1,6510^{-2} \mathrm{~Sv}^{-1}$ concernant les effets graves sur la santé; ce facteur de risque pour toute la vie fonde les recommandations de base actuelles de la CIPR et se déduit d'un modèle de risque absolu. Ce risque est d'environ $1 \%$ pour $10 \mathrm{mSv}$ par an. A sa réunion de Como, en septembre 1987, la CIPR, cependant, s'est interrogée sur la question des estimations de risque [2]; elle a conclu que les résultats d'une étude définitive des nouveaux calculs de doses reçues par les survivants des explosions atomiques, couplés à un temps de suivi plus long, pourrait augmenter l'estimation du risque d'un facteur deux, approximativement. Un rapport de la CIPR sur le risque de cancer du poumon dû aux descendants du radon était également publié en août 1987 ; on y adoptait un modèle de risque relatif pour extrapoler à l'ensemble de la population des données épidémiologiques concernant les mineurs [3]. Pour une population de référence définie par l'espérance de vie et la fréquence "sous-jacente"1 de cancers du poumon, on a estimé le risque sur la vie à 1,3\% environ, pour une exposition continue à une concentration ERE de $100 \mathrm{~Bq} \mathrm{~m}^{-3}$, ou à $10 \mathrm{mSv}$ par an. Pour la population de la Grande-Bretagne, le risque sur la vie serait approximativement doublé. Cette récente évolution suggère donc que le risque lié à l'exposition aux descendants du radon, dans les habitations, peut être le double au moins de celui auquel conduisaient les positions de base de la CIPR, il y a environ un an.

On peut évaluer la signification potentielle pour la santé, en GrandeBretagne, de l'exposition continue aux descendants du radon, en effectuant une comparaison avec les autres causes de décès prématurés [5]. Comme l'a fait la CIPR, une telle comparaison des risques a beaucoup pesé dans la manière dont le NRPB a considéré cette question.

\section{LES RECOMMANDATIONS DU NRPB}

Voici l'essentiel des recommandations du NRPB:

"II faut intervenir dans certaines habitations existantes pour réduire l'exposition au radon, et il faut prendre des mesures pour limiter l'exposition dans les habitations à venir. Le schéma suggéré par la CIPR est une base de départ appropriée. Prenant en compte des facteurs tels que le niveau relatif de risque, la faisabilité et le coût de la mise en œuvre des mesures à prendre, le nombre d'habitations concernées, le NRPB donne les recommandations suivantes concernant le Royaume-Uni:

"Pour les habitations existantes, on recommande de prendre des mesures graduelles, en fonction de l'équivalent de dose efficace, au-dessus d'un niveau d'action de $20 \mathrm{mSv}$ par an. A ce niveau d'action, on doit s'en tenir à des mesures simples pour réduire l'exposition au radon.

1. Risque pour la population s'il n'y avait pas le radon. 
A un niveau nettement plus élevé, il est probable qu'il s'avèrera nécessaire d'intervenir énergiquement. On devra aussi avoir connaissance de l'importance de la dose annuelle dans certains cas particuliers, pour décider de l'urgence de l'action à effectuer: on devra donner priorité aux habitations où les niveaux sont les plus élevés.

"Pour les habitations futures, on recommande une limite supérieure de $5 \mathrm{mSv}$ par an comme base pour introduire des modifications aux procédés de construction, dans les régions où des niveaux élevés de radon sont probables".

Les recommandations du NRPB ont introduit des modalités de délai que l'on ne trouve pas dans les recommandations de la CIPR [1]: l'importance de la dose annuelle doit déterminer l'urgence de l'action qui doit conduire à la diminuer. La RCEP a approuvé ce principe, en recommandant que l'on donne la priorité aux habitations où les niveaux sont préoccupants [6]. II a été également reconnu que le point primordial est l'exposition prolongée, durant de nombreuses années, à des niveaux élevés. Etant donné la diversité des situations que l'on doit s'attendre à trouver, il a semblé déraisonnable d'édicter une règle stricte; on a donné un guide permettant d'établir la ligne de conduite à adopter. Pour toutes les doses qui se situent au-dessus du niveau d'action, on doit agir aussi vite que cela est raisonnablement possible, et, pour des doses annuelles au-dessus de $50 \mathrm{mSv}$ environ, on doit intervenir dans un délai d'un an environ. Pour des doses annuelles comprises entre $20 \mathrm{mSv}$ et $50 \mathrm{mSv}$, il serait souhaitable que l'on intervienne avant quelques années. Dans chaque cas, le délai doit courir depuis le moment où des mesures appropriées auront confirmé l'existence de niveaux élevés.

\section{NOMBRE D'HABITATIONS}

Le NRPB a mesuré le radon dans 2300 habitations choisies au hasard sur l'ensemble du territoire du Royaume-Uni; ces résultats ont été complétés par des mesures dans 700 habitations situées dans des zones où les caractéristiques géologiques sont de nature à créer, à l'intérieur des habitations, des niveaux de radon élevés, principalement dans le sud-ouest de l'Angleterre [7]. La moyenne géométrique de l'équivalent de dose efficace annuel dû aux descendants du radon dans les habitations est de 0,7 mSv environ, mais il y a une grande dispersion des résultats. L'analyse des résultats a montré que, sur un ensemble de 21 millions d'habitations, le nombre approximatif de celles où la dose annuelle dépasse une valeur de référence donnée, augmente très rapidement quand on diminue cette valeur. De même les zones où l'on voit apparaître un nombre appréciable d'habitations avec des doses supérieures à la valeur de référence, augmentent rapidement quand diminue cette valeur. II y a aussi des problèmes pratiques pour identifier les habitations qui se situent au-dessus des valeurs de référence: leur nombre augmente très vite lorsque l'on diminue cette valeur; cela est dû principalement à ce que l'on ne peut définir clairement les zones géographiques, et à ce que le nombre total des habitations qu'il est nécessaire de surveiller pour en trouver au-dessus de la valeur de référence 
devient beaucoup plus grand. Cependant, avec le niveau d'action adopté, la majeure partie des 20000 habitations situées aux niveaux les plus élevés devraient se trouver dans le sud-ouest de l'Angleterre, en général dans une région granitique, ou au voisinage. On a jugé qu'il fallait donner la priorité à ces régions.

On estime que parmi les habitations nouvelles construites chaque année, quelques milliers devraient dépasser la limite supérieure de $5 \mathrm{mSv}$ par an. On devrait trouver la plupart dans les mêmes régions de l'Angleterre du sudouest. Au Royaume-Uni, la cadence à long terme de renouvellement des habitations est d'environ 200000 par an, bien que cette valeur fluctue beaucoup selon les conditions économiques.

Donc, au total, $0,1 \%$ des habitations existantes seraient susceptibles d'être concernées par ce niveau d'action, alors que $1 \%$ des constructions nouvelles relèveraient de la limite supérieure. Enfin, l'impact géographique est globalement bien délimité.

\section{MESURES A PRENDRE}

La cause des doses élevées, dues au descendants du radon présents dans les habitations, est le taux d'entrée avec lequel le radon gazeux venant du sol y pénètre. L'augmentation de la ventilation peut réduire la concentration du radon; cependant, l'action la plus appropriée consiste à diminuer le transfert du radon vers l'espace habité, soit en faisant du plancher une barrière plus efficace, soit en éloignant le gaz de l'espace situé au-dessous du plancher.

Pour les habitations existantes, on estime grossièrement que les coûts se situent entre 100 livres pour une intervention simple, comme le scellement des fissures du plancher, en passant par 1000 livres pour une intervention d'importance moyenne, comme l'extraction du gaz au-dessous du plancher, et jusqu'à 10000 livres pour une action importante telle que le remplacement du plancher. II serait donc possible de choisir des interventions proportionnées aux doses. En ce qui concerne les constructions nouvelles, on pourrait adopter des techniques de construction appropriées qui, sans briser avec les pratiques actuelles, permettraient d'éviter des expositions élevées pour les futurs occupants.

De telles modifications peuvent entraîner des coûts additionnels pas trop importants.

\section{L'ACTION GOUVERNEMENTALE}

Le gouvernement a accepté cette recommandation; le ministre d'État pour l'environnement a fait un exposé sur le radon devant la Chambre des Communes, et il a annoncé un programme de travail pour s'atteler à ce 
problème. Ce programme présente deux parties: l'identification des maisons où les concentrations en radon sont au-dessus du niveau d'action d'une part, le déploiement des mesures curatives et préventives, d'autre part.

On peut identifier les maisons se situant au-dessus du niveau d'action par trois voies distinctes, mais liées les unes aux autres:

- des contrôles systématiques, portant sur une année entière, de toutes les habitations immédiatement voisines des maisons présentant les concentrations en radon les plus élevées;

- des mesures faites à la demande, aux frais du gouvernement, dans les régions où les niveaux potentiels de radon peuvent être élevés;

- des mesures faites à la demande, aux frais des propriétaires, dans les autres régions.

Les contrôles ont commencé durant l'été 1987 dans le sud-ouest de l'Angleterre. Les premiers résultats confirment les estimations du nombre d'habitations relevant du niveau d'action.

Le gouvernement finance aussi un programme de recherche permettant de développer les mesures curatives et préventives. Cela conduira à des notes d'orientation sur les mesures à prendre contre le radon, à l'intention des propriétaires et des constructeurs. En ce qui concerne l'importante question des coûts, le ministre a fait le commentaire suivant:

"La responsabilité des mesures curatives doit relever du propriétaire de la maison ou du terrain dans le cas de location tant publique que privée. Le gouvernement est disposé à étudier des subventions sur le coût des travaux curatifs dans le cas des propriétaires-occupants qui en ont le plus besoin".

\section{CONCLUSION}

On s'accorde en général sur le point que les descendants du radon sont, en moyenne, la cause principale de l'exposition de l'homme aux rayonnements ionisants: avec les descendants du thoron, ils représentent plus de $50 \%$ du total. C'est dans la nature de la vie, mais on s'est attelé, d'une manière judicieuse et avec mesure, au problème de l'exposition excessive aux descendants du radon dans les habitations de l'ensemble du Royaume-Uni. On a délimité le problème, on a établi des niveaux d'action, on est en train de faire des enquêtes pour identifier les maisons particulièrement à risque, et l'on met en place des mesures curatives et préventives. 


\section{BIBLIOGRAPHIE}

[1] INTERNATIONAL COMMISSION ON RADIOLOGICAL PROTECTION (ICRP). Principales for limiting exposure of the public to natural sources of radiation, ICRP publication 39. Oxford: Pergamon press, 1987.

[2] INTERNATIONAL COMMISSION ON RADIOLOGICAL PROTECTION (ICRP). Statement from the 1987 Como meeting. Radiol. Prot. Bull., 1987, suppl. to N.86, et Radioprotection, 1987, 23 (1) 65-77.

[3] INTERNATIONAL COMMISSION ON RADIOLOGICAL PROTECTION (ICRP). Lung cancer risk from indoor exposures to radon daughters, ICRP publication 50. Oxford: Pergamon press, 1987.

[4] NATIONAL RADIOLOGICAL PROTECTION BOARD (NRPB). Exposure to radon daughters in dwellings. Rapport ASP-10, 1987.

[5] NATIONAL RADIOLOGICAL PROTECTION BOARD (NRPB). Exposure to radon daughters in dwellings. Rapport NRPB-GS-6, 1987.

[6] ROYAL COMMISSION ON ENVIRONMENTAL POLLUTION (RCEP). Tackling pollution - experience and prospects (Cmnd 9149). Londres: HMSO, 1984.

[7] WRIXON A.D. et coll. Natural radiation exposure in UK dwellings. Rapport NRPB-R 190, 1987. 